\title{
Tratamento cirúrgico das lesões traumáticas do plexo braquial
}

\author{
Daniella Brito Rodrigues' ${ }^{1}$ Maria Luana Carvalho Viegas², Juvenal \\ de Souza Rogério ${ }^{3}$,Edmundo Luis Rodrigues Pereira ${ }^{3}$
}

Universidade do Estado do Pará (UEPA), Hospital Metropolitano de Urgências e Emergências (HMUE), Belém, PA, Brasil.

\section{RESUMO}

As lesões do plexo braquial são frequentemente causadas pela avulsão de raízes nervosas após acidentes automobilísticos de alta energia e geralmente afetam homens jovens, resultando em déficits neurais com elevado potencial de incapacidade grave e permanente. O presente estudo tem como objetivo revisar a literatura pertinente ao tratamento cirúrgico das lesões traumáticas do plexo braquial, ressaltando as principais técnicas e indicações. São discutidas as quatro técnicas cirúrgicas mais comumente utilizadas: reparação direta nas lacerações agudas, neurólise externa, enxertos de nervos e transferência de nervos. Além disso, são analisados a anatomia, a fisiopatologia e os fatores que influenciam a recuperação funcional: idade, intervalo entre a lesão e cirurgia, estado dos dois sítios de coaptação, enxertos de nervos longos, tecido cicatricial, isquemia e grau de lesão da raiz nervosa. A reparação direta é mais utilizada em lacerações agudas, e a transferência de nervos tem se mostrado a técnica cirúrgica mais eficaz, principalmente em combinação com os enxertos de nervos.

\section{PALAVRAS-CHAVE}

Plexo braquial, transferência de nervo, traumatismos dos nervos periféricos, plexo braquial/cirurgia.

\section{ABSTRACT}

Surgical treatment of traumatic brachial plexus injuries

The brachial plexus injuries are frequent caused by nerve roots avulsion after high-energy traffic accidents and generally affect young males, resulting in neural deficits with high potential for permanent disability. The present study has an objective to perform a review the literature concerning the surgical treatment of traumatic lesions of the brachial plexus, emphasizing the key techniques and indications. It discusses the four most commonly used surgical techniques: direct repair in acute lacerations, external neurolysis, nerve grafts and nerve transfers. Furthermore, analyzes the anatomy, pathophysiology and the factors that influence the functional recovery: age, the interval between injury and surgery, the state of coaptation of the two sites, nerve grafts, scarring, ischemia and the degree of lesion nerve root. $A$ direct reparation is most used in acute lacerations and the nerve transfer have been shown the most effective treatment, especially in combination with the nerve grafts.

\section{KEYWORDS}

Brachial plexus, nerve transfer, peripheral nerve injuries, brachial plexus/surgery.

1 Acadêmica de Medicina da Universidade do Estado do Pará (UEPA), Belém, PA, Brasil.

2 Residente de Neurocirurgia do Hospital Ophir Loyola, Belém, PA, Brasil.

3 Neurocirurgião do Hospital Metropolitano de Urgências e Emergências (HMUE), Belém, PA, Brasil. 


\section{Introdução}

O plexo braquial é um conjunto de nervos que emergem da medula espinhal cervical entre C5 e T1. Responsáveis pelos movimentos e sensibilidade dos membros superiores, frequentemente sofrem lesões por estiramento ou avulsão durante acidentes automobilísticos, levando a déficits neurais significativos, com elevado potencial de sequelas definitivas. ${ }^{1-5} \mathrm{O}$ manejo de tais lesões requer conhecimento apropriado de anatomia, fisiologia e da história natural dessas lesões, cuja frequência tende a aumentar proporcionalmente ao crescimento de vias públicas e do uso de veículos automotores. ${ }^{4-7}$

\section{Anatomia}

Responsáveis pela inervação dos membros superiores, suas raízes formam três troncos: superior (C5 e C6), médio (C7) e inferior (C8 e T1). Tais troncos estão localizados na fossa supraclavicular e dividem-se em dois ramos, um anterior e um posterior, que, por sua vez, formam os fascículos, situados em torno da artéria axilar, a qual está associada também à nomenclatura desses fascículos, isto é, fascículo lateral, porque está situado lateralmente à artéria axilar. Os ramos anteriores dos troncos superior e médio formam o fascículo lateral; o ramo anterior do tronco inferior forma o fascículo medial; e os ramos posteriores dos três troncos formam o fascículo posterior. Esses fascículos se subdividem nos ramos terminais (nervo radial, nervo axilar, nervo mediano, nervo ulnar e nervo músculo-cutâneo) do plexo braquial na borda inferior e lateral do músculo peitoral menor. ${ }^{8}$

\section{Fascículo lateral}

Transporta fibras originalmente de C5 até C7 e possui três ramos: um ramo lateral, que origina o nervo peitoral lateral, e dois ramos terminais, que originam o nervo musculocutâneo e a raiz lateral do nervo mediano. O nervo musculocutâneo supre todos os músculos do compartimento anterior do braço (coracobraquial, bíceps braquial e braquial) e continua como nervo cutâneo lateral do antebraço; sua lesão está associada com a abolição do reflexo bicipital.

\section{Fascículo posterior}

Conduz as fibras de $\mathrm{C} 5$ a T1, possui cinco ramos: três ramos laterais que formam os nervos subescapular, toracodorsal e subescapular inferior e dois ramos terminais que originam os nervos axilar e radial.

$\mathrm{O}$ nervo axilar recebe fibras de $\mathrm{C} 5$ e C6, tem como trajeto a face posterior do braço juntamente com a artéria circunflexa posterior do úmero, através do espaço quadrangular, origina o nervo cutâneo lateral do braço e é responsável pela inervação dos músculos redondo menor e deltoide.

O nervo radial é o maior ramo do plexo braquial, recebe fibras provenientes de $\mathrm{C} 5$ a $\mathrm{T} 1$, tem trajeto posterior à artéria axilar e anterior aos músculos subescapular, redondo maior e latíssimo do dorso; percorre o sulco do nervo radial do úmero juntamente com a artéria braquial profunda, onde fica vulnerável à lesão quando há fratura de úmero. Inerva os músculos tríceps braquial, braquiorradial e extensores do antebraço.

\section{Fascículo medial}

Conduz as fibras de $\mathrm{C} 8$ e $\mathrm{T} 1$ e possui cinco ramos: três ramos laterais que originam os nervos peitoral medial, cutâneo medial do braço e cutâneo medial do antebraço e dois ramos terminais que dão origem ao nervo ulnar e à raiz medial do nervo mediano.

$\mathrm{O}$ nervo ulnar recebe fibras de $\mathrm{C} 8 \mathrm{e} \mathrm{T} 1$, às vezes, de C7 também; tem como trajeto a face medial do braço, onde não emite nenhum ramo, e depois percorre posteriormente o epicôndilo medial para entrar no antebraço. Inerva os músculos flexor ulnar do carpo, metade ulnar do flexor profundo dos dedos, adutor do polegar e parte profunda do flexor curto do polegar, músculos da região hipotenar, terceiro e quarto lumbricais e todos os interósseos.

O nervo mediano é lateral à artéria axilar e é formado por duas raízes (lateral e medial), originadas do fascículo lateral e do fascículo medial. As fibras que se originam do cordão lateral inervam a sensibilidade da eminência tenar (C6), polegar (C6), indicador (C6-C7) e dedo médio (C7) e a motricidade dos músculos pronador redondo e flexor radial do carpo. As fibras que se originam do cordão medial inervam a sensibilidade da metade lateral do dedo anelar (C7-C8) e a motricidade de todos os outros músculos inervados pelo mediano. O nervo mediano emite diversos ramos ao longo de seu trajeto, o nervo interósseo anterior é um ramo exclusivamente motor do nervo mediano, responsável pela inervação dos músculos flexor profundo para o II e III dedos, flexor longo do polegar e pronador quadrado; seu primeiro ramo sensorial é o nervo cutâneo palmar emitido no antebraço distal, este passa externamente ao túnel do carpo para inervar a eminência tenar na face palmar da mão. Após cruzar o túnel do carpo, o nervo mediano inerva através de seu ramo recorrente tenar os músculos abdutor curto do polegar, oponente 
do polegar e a porção superficial do flexor curto do polegar. ${ }^{8}$ Além disso, o nervo mediano emite ramos digitais sensoriais terminais para a face palmar do polegar, indicador, dedo médio e metade lateral do dedo anelar e inerva a porção dorsal das falanges distais dos dedos indicador e anelar.

\section{Classificação das lesões}

Para Seddon, ${ }^{9}$ as lesões nervosas podem ser em três níveis: na neuropraxia há preservação da estrutura do nervo, mas pode haver paralisia com perda temporária da função motora e preservação da sensibilidade; a axonotmese apresenta degeneração walleriana com ruptura axonal, podendo haver nela recuperação conforme o nível da lesão; a mais grave é a neurotmese, na qual ocorre a descontinuidade da estrutura nervosa, não ocorrendo recuperação espontânea e requerendo intervenção cirúrgica.

Ademais, lesões do plexo podem ser divididas em aberta, fechada (tração), após radiação e paralisia perinatal..$^{10}$ Quanto mais energia tiver o trauma, maiores serão as lesões e estruturas anatômicas envolvidas como vasos, tendões e ossos. ${ }^{1-3}$ As lesões abertas geralmente estão associadas a múltiplas fraturas ósseas e articulares e requerem intervenção cirúrgica imediata; as lesões fechadas são as mais frequentes e estão comumente relacionadas a traumas de alta energia como acidentes motociclísticos; enquanto a paralisia perinatal do plexo braquial mais comum é a superior de Erb, que é uma lesão obstétrica do tronco superior C5-C6 e possui resolução espontânea em grande parte dos casos. ${ }^{2,10}$ As lesões de piores prognósticos são aquelas que se comunicam o gânglio estrelado e as de avulsão radicular da medula, em que a dor é incontrolável e de difícil manejo; o sinal de Horner é identificado por miose, anidrose, enoftalmia e ptose palpebral ipsilateral, por avulsão de C8-T1. Entretanto, a presença do sinal de Tinel, isto é, choque à percussão digital sobre o trajeto anatômico do nervo, indica lesão mais periférica com melhor prognóstico. ${ }^{11}$

\section{Epidemiologia}

Diversos autores apontaram como características epidemiológicas importantes: acometimento de indivíduos jovens, decorrentes de mecanismos de tração sobre as estruturas nervosas, sendo os acidentes motociclísticos os mais envolvidos, com avulsão radicular como principal mecanismo de injúria., ${ }^{2,45}$ Moran et al. ${ }^{6}$ destacam como aspecto epidemiológico o acometimento de homens, com idade entre 15 e 25 anos; autores como Narakas et al., ${ }^{7}$ após 10 anos de experiência, analisaram que $70 \%$ das lesões do plexo braquial são secundárias a acidentes de veículos, e desses, $70 \%$ terão outras lesões graves associadas. Em nosso país, Silva et $a .^{2}{ }^{2}$ consideram os acidentes motociclísticos como os principais responsáveis pelo aumento do número de pacientes com essa patologia, devido ao número crescente de motociclistas.

\section{Indicações para o tratamento cirúrgico}

Cerca de dois terços dos pacientes com lesão do plexo braquial têm recuperação espontânea ao longo dos primeiros meses, portanto a necessidade de tratamento cirúrgico dependerá do grau de recuperação do paciente, devendo somente ser realizado na ausência de evidência clínica ou eletrofisiologicamente comprovada de recuperação ou quando a recuperação espontânea for impossível por causa a avulsões mais graves como a neurotmese. ${ }^{12}$ Teoricamente, todos os pacientes sem recuperação espontânea podem se beneficiar da intervenção microcirúrgica do plexo braquial. Os principais objetivos da cirurgia para lesão do plexo braquial são a restauração dos movimentos e a resolução de dor. ${ }^{13}$

Para Siqueira e Martins ${ }^{14}$, o tratamento cirúrgico deve estabelecer como prioridades: preservação do movimento de flexão de cotovelo, estabilização do ombro e consequente recuperação de abdução e rotação externa, porque um ombro instável impedirá o uso da extremidade; a seguir, a extensão do cotovelo, a abdução do braço contra o peito, extensão do dedo e do pulso e, por último, as estruturas inervadas pelo nervo ulnar, por causa do mau prognóstico funcional. Além disso, devem-se priorizar os músculos mais proximais, porque eles têm maior capacidade de reinervação em comparação com os mais distais. ${ }^{12}$

Para diversos autores ${ }^{13-17}$ a intervenção cirúrgica ideal deve ser realizada entre o terceiro e o quarto mês após o trauma, quando se pode observar se há recuperação espontânea ou se a lesão é irreversível, além de ser um tempo adequado para a recuperação dos músculos que serão reinervados; do sexto ao décimo segundo mês a cirurgia ainda é possível, mas os resultados pioram progressivamente, e depois de um ano a cirurgia só é realizada em casos especiais. Consenso, entretanto, é que o melhor momento para a intervenção cirúrgica depende do mecanismo da lesão. 


\section{Cirurgia na fase aguda}

Cirurgia precoce é indicada principalmente em lesões abertas, em pacientes com forte evidência de uma lesão pré-ganglionar, com paralisia total, aumento do déficit neurológico, em que há suspeita de hematoma e fístula, ferimentos por arma de fogo e/ou lesões vasculares graves associadas. A primeira conduta para as lesões do plexo por estiramento é a observação clínica. Deve-se aguardar a possível recuperação espontânea, sempre com acompanhamento de fisioterapia e avaliação eletrofisiológica periódica, entretanto o seguimento clínico prolongado tem seus riscos, como o desenvolvimento de atrofia muscular; o contrário acontece nas lesões abertas dilacerantes, que devem ser operadas dentro das primeiras 72 horas. ${ }^{18}$ A reparação direta por meio da neurorrafia término-terminal é a principal técnica utilizada nas lacerações agudas do plexo braquial.

\section{Técnicas cirúrgicas}

A via anterior é a mais comumente utilizada para a cirurgia do plexo braquial e, dependendo do tipo e da extensão da lesão, a abordagem pode ser supraclavicular, infraclavicular ou combinada. Quatro técnicas cirúrgicas são mais comumente utilizadas:

\section{Reparação direta}

Por meio da neurorrafia término-terminal sem tensão na linha de sutura. É geralmente utilizada em lacerações agudas, entretanto o retorno da função não pode ser garantido, especialmente para alvos desnervados que estão mais distantes da localização primária da lesão. Entretanto, é considerada eficaz em reparos primários para lacerações, com prognóstico favorável, principalmente em lesões parciais, desde que seja estabelecida precocemente. ${ }^{18,19}$

\section{Neurólise externa}

É a liberação de nervos intactos do tecido cicatricial, retirando as aderências fibróticas em torno do nervo; a neurólise é indicada quando há intensa reação fibrótica perineural, geralmente desencadeada por hematoma pós-traumático ou trauma por estiramento. ${ }^{19-22} \mathrm{Seg}$ mentos de nervos são libertados circunferencialmente e nas direções proximal e distal de cada lado do segmento lesado. É considerada por alguns autores ${ }^{22}$ como um procedimento controverso, cujos resultados são incertos, mas que pode ser indicado em casos de neu- ropraxia, se esta não se resolver espontaneamente ou se a resolução for demasiado lenta. É importante ressaltar que a neurólise pode ser realizada em todas as cirurgias em que se identifica reação fibrótica perineural, isolada, ou associada às demais técnicas. A neurólise interna é um procedimento raro, de difícil execução, com resultados incertos.

\section{Enxerto de nervo}

Quando há uma ruptura grave pós-ganglionar ou uma lesão em continuidade, o elemento lesionado deve ser ressecado. A grande diferença interneural geralmente persiste, e os cotos retraídos não podem ser reunidos sem tensão significativa. Nessa situação, a interposição de autoenxertos é necessária, a fim de reduzir os cotos e permitir o reparo fascicular quando há perda de substância e descontinuidade anatômica. ${ }^{2}$ Entretanto, colas biológicas como tissucol têm sido cada vez mais utilizadas. ${ }^{23} \mathrm{Na}$ maioria dos enxertos, é utilizado o nervo sural. Os enxertos podem ser vascularizados ou livres, curtos ou longos. Enxertos curtos (5-7 cm de comprimento) são usados para reparar lesões menores, do tronco superior; enquanto lesões extensas necessitam de enxertos mais longos $(10-15 \mathrm{~cm})$; enxertos vascularizados têm apresentado bons resultados e são utilizados para facilitar a progressão axonal, principalmente em lesões extensas. ${ }^{24,25}$

\section{Transferência de nervo/neurotização}

A utilização de um nervo íntegro como fonte de axônios para reinervação de elementos distais do plexo braquial modificou os resultados pós-operatórios em lesões graves e até nas avulsões de raízes nervosas. ${ }^{26,27}$ Pode ser feita por meio da transferência simples de nervos de fora do plexo ou oriundos do plexo, isto é, intra ou extraplexuais, ou até por meio da interposição de enxertos. ${ }^{28}$ Tradicionalmente, as transferências nervosas foram reservadas para reparação de lesões irreparáveis por avulsão do plexo braquial, para o tratamento de lesões pré-ganglionares. ${ }^{29}$ Entretanto, por causar redução no tempo necessário para estabelecer a reinervação, diminuindo a distância entre o local da reparação do nervo e do órgão final, sua indicação inicial foi expandida de tal modo que agora é também usada para os casos de recuperação lenta. ${ }^{30,31}$ Os nervos mais comuns para a transferência incluem o acessório espinhal distal, nervo intercostal e nervo peitoral medial; os fascículos funcionantes do mediano e ulnar, ramos do nervo radial e frênico, cervical profundo e o hipoglosso têm sido utilizados menos comumente. ${ }^{14} \mathrm{~A}$ transferência de nervos para a raiz ventral da medula espinhal é um procedimento que pode ser realizado quando há avulsões proximais completas. 


\section{Discussão}

O tratamento cirúrgico para as lesões do plexo braquial é complexo e exige avaliações minuciosas relacionadas ao mecanismo de trauma e a possível recuperação funcional para a escolha da técnica mais adequada. ${ }^{32}$ Muitos fatores influenciam a recuperação funcional após o tratamento cirúrgico de lesões do plexo braquial, tais como a idade do paciente, o intervalo entre a lesão e a cirurgia (período de desnervação), o estado dos dois sítios de coaptação, enxertos de nervos longos, tecido cicatricial, isquemia e o grau de lesão da raiz nervosa. ${ }^{2,14}$ Pacientes jovens, com períodos curtos de denervação (menos de seis meses) e lesões pós-ganglionares, geralmente apresentam melhor resultado do que pacientes com avulsão de raiz medular. Dor persistente (com duração de mais de seis meses) é um sinal de mau prognóstico para a recuperação neurológica, independentemente da localização. ${ }^{33-35}$

Kandenwein et al. ${ }^{36}$ acreditam que as lesões traumáticas do plexo braquial têm resultado geralmente satisfatório, do ponto de vista funcional, especialmente quando realizadas entre três e cinco meses após o trauma, e consideram que a escolha das diferentes técnicas cirúrgicas deve ser adaptada de forma individualizada para o paciente e que o monitoramento eletrofisiológico e o acompanhamento intensivo com a fisioterapia tanto no pré quanto no pós-operatório são essenciais para a recuperação. $\mathrm{O}$ tratamento cirúrgico das lesões crônicas é demasiado controverso, e há poucos relatos de bons resultados nesta fase; entretanto, Souza et al. ${ }^{37}$ avaliaram os resultados da transposição do tríceps para a flexão do cotovelo em pacientes portadores de lesão crônica e completa do tronco superior do plexo braquial e encontraram que a transposição anterior do músculo tríceps proporcionou satisfação dos pacientes em todos os casos e que essa técnica é viável na lesão crônica do tronco superior do plexo braquial.

Haninec et al. ${ }^{13}$ destacam que, apesar das evoluções no contexto das técnicas microcirúrgicas, os resultados são considerados favoráveis somente em $60 \%$ dos pacientes. Esses autores ressaltam, ainda, a dificuldade no tratamento daqueles pacientes com avulsão de raizes nervosas, porque a reparação direta é impossível e a neurotização é a base do tratamento, persistindo a discussão acerca de quais nervos devem ser utilizados, tendo em vista os resultados controversos. De modo geral, eles consideram que os resultados da reparação direta do plexo braquial são melhores do que os resultados da neurotização. Para Gepp et al., ${ }^{5}$ a realização de transferências nervosas modificou o tratamento cirúrgico das lesões graves, com avulsão de raízes nervosas, e é considerada a forma preferencial de tratamento das lesões de plexo braquial para vários autores.
Para Flores ${ }^{1}$, o tratamento cirúrgico possui resultados geralmente satisfatórios para reinervação da musculatura proximal do membro superior, no entanto os mesmos bons resultados não são observados na reinervação da mão. A técnica de enxerto de nervo espinhal proximal, assim como a transferência de nervo, é eficaz para a reinervação do plexo braquial; no entanto, a associação de transferência e reparo do nervo, assim como a transferência de tendão para a reabilitação da mão, é a melhor alternativa para aumentar a recuperação funcional após paralisia total. Disso discordam autores como Tung e Mackinnon, ${ }^{38}$ que defendem a técnica cirúrgica restrita a transferência de nervo, devendo-se evitar a associação com enxerto de nervo. Siqueira e Martins ${ }^{14}$ concordam com Flores ${ }^{1}$ quanto aos melhores resultados obtidos com a associação entre enxertos e transferências de nervos; similarmente, a reinervação dos músculos intrínsecos da mão não é esperada pelas técnicas convencionais, enquanto a reinervação sensorial do nervo mediano pode ser conseguida em até $80 \%$ dos casos. A questão primordial não é a estratégia escolhida, se simples ou combinada, e sim que a obtenção de melhores resultados cirúrgicos parece ser determinada pelo potencial de um nervo doado como uma fonte de axônios motores. ${ }^{1}$

Sulaiman et $a l .{ }^{26}$, ao estudarem durante 10 anos os resultados de transferência de nervos, na Universidade de Louisiana, concluíram que a maior vantagem da transferência de nervo em comparação com o enxerto é a capacidade que a transferência tem de converter uma lesão nervosa de elevada gravidade em uma lesão nervosa de menor gravidade, com maior recuperação funcional, principalmente para a flexão do cotovelo e a abdução do ombro, principais objetivos da cirurgia. Para esses autores, a transferência de nervos é mais eficaz do que os enxertos, e a reparação cirúrgica direta deverá ser realizada sempre que possível e pode ser associada com os procedimentos de transferência de nervo, dependendo do grau da lesão.

Oberlin et al. ${ }^{39}$ publicaram uma técnica de neurotização que consiste na dissecção de dois fascículos do nervo ulnar, que são anastomosados no nervo motor do bíceps, ramo do nervo musculocutâneo lesado, para restauração da flexão do cotovelo, e tem apresentado resultados satisfatórios no pós-operatório quanto à recuperação de flexão do cotovelo, sem déficit funcional da mão pela dissecção do nervo ulnar. Para Sedain et al. ${ }^{27}$, a transferência de nervo distal (Oberlin) pode ser uma opção valiosa para restaurar a função do bíceps em pacientes cujo tratamento será tardio, ou após falhas no tratamento por reparação direta e enxertos. A recuperação funcional pôde ser observada em $78 \%$ dos pacientes operados após seis meses de lesão. Para esses autores, as transferências de Oberlin obtiveram 
uma taxa de sucesso de $100 \%$ nos pacientes operados dentro de 12 meses após a lesão. A técnica conhecida como Oberlin II disseca fascículos dos nervos ulnar e mediano e transfere-os aos ramos motores dos músculos bíceps e braquial, o que potencializa a flexão do cotovelo devido à recuperação do músculo braquial. Essa técnica foi considerada como procedimento-padrão nas lesões altas do plexo braquial em pacientes com até seis meses de lesão. ${ }^{40,41}$

Batista e Araújo $^{18}$ consideram que as técnicas de reparo com neurorrafia término-terminal epineural e a interposição de enxertos de nervo são as que apresentam os melhores resultados. Ainda que os resultados do emprego de enxertos não sejam totalmente satisfatórios, essa técnica é considerada por alguns autores ${ }^{42,43}$ como padrão-ouro das reconstruções tardias, devido à baixa morbidade. Martins et al. ${ }^{44}$ apresentaram uma técnica de transferência de nervo utilizando um novo fascículo saudável do cordão posterior para a reconstrução do nervo supraescapular e obtiveram bons resultados. Eles concluíram que essa nova transferência de nervo intraplexual poderia ser aplicada em pacientes com lesão isolada do tronco superior e lesão concomitante dos nervos doadores extraplexuais normalmente utilizados para a reinervação do nervo supraescapular.

Para Siqueira e Martins, ${ }^{14}$ o enxerto de nervo apresenta bons resultados em $75 \%$ das lesões supraclaviculares. Entre as lesões infraclaviculares, o bom prognóstico do enxerto de nervo depende da proximidade do nervo, isto é, o nervo musculocutâneo, com o músculo desinervado, podendo-se obter resultados favoráveis em até $80 \%$ dos casos, enquanto as lesões das raizes do tronco médio e inferior apresentam prognóstico mais desfavorável. Para esses autores, a reinervação do punho e do flexor digital e extensor pode ser esperada em 50\%-60\% dos casos, mas a reinervação dos músculos intrínsecos da mão não é um resultado esperado. Embora possa haver retorno dos movimentos do punho e do dedo, a restauração funcional da mão ainda é rara. Além disso, esses autores também consideram a utilização de combinações de enxertos de nervos e transferências nervosas como uma opção terapêutica importante, atrelada a resultados mais satisfatórios.

\section{Considerações finais}

As lesões do plexo braquial possuem indicação cirúrgica precoce restrita, sendo a maior parte dos casos reservada para tratamento cirúrgico entre três e quatro meses após o trauma. Esse tratamento possui diversas técnicas, sendo a reparação direta mais utilizada em lacerações agudas, em tratamentos cirúrgicos precoces em politraumatizados. A transferência de nervo tem se mostrado a mais eficaz nas intervenções tardias, principalmente em combinação com os enxertos de nervos. A transferência de Oberlin é uma importante ferramenta em cirurgias seis meses após o evento traumático; entretanto, a recuperação funcional da mão ainda é deficiente após essas técnicas cirúrgicas, sendo indicada a transferência de tendão nesses casos.

\section{Referências}

1. Flores LP. Epidemiological study of the traumatic brachial plexus injuries in adults. Arq Neuropsiquiatr. 2006;64(1):8894.

2. Silva JLB, Silva PG, Gazzalle A. Lesões do plexo braquial. Rev AMRIGS. 2010;54(3):344-9.

3. Yeng LT, Kasiyama HHS, Teixeira MJ. Síndromes dolorosas dos membros superiores. Rev Med. 2001;80:317-34.

4. Mélega JM, editor. Cirurgia plástica fundamentos e arte: cirurgia reparadora de troncos e membros. Rio de Janeiro: Guanabara-Koogan; 2004.

5. Gepp RA, Couto JMC, Cardoso MM, Neri EA, Silva RT. Abordagem posterior para transferência nervosa do nervo acessório para o nervo supraescapular. Arq Bras Neurocir. 2011;30(3):89-93.

6. Moran SL, Steinmann SP, Shin AY. Adult brachial plexus injuries: mechanism, patterns of injury, and physical diagnosis. Hand Clin. 2005;21(1):13-24.

7. Narakas A, Allieu Y, Alnot JY. Neurotisation par le nerf spinal dansles lesions du plexus brachial. In: Alnot JY, Narakas A editors. Les paralysies du plexus brachial. Paris: Expansion Scientifique Française; 1989. p. 191-9.

8. Moore KL, Dalley II, Agur AF, Anne MR. Anatomia orientada para a clínica. $6^{\mathrm{a}}$ ed. Rio de Janeiro: Guanabara Koogan; 2011.

9. Seddon HJ. Surgical disorders of the peripheral nerves. 2nd ed. Edinburgh: Churchill Livingstone; 1975.

10. Kim DH, Murovic JA, Tiel RL, Kline DG. Mechanisms of injury in operative brachial plexus lesions. Neurosurg Focus. 2004;16(5):E2.

11. Andersen J, Watt J, Olson J, Van Aerde J. Perinatal brachial plexus palsy. Paediatr Child Health. 2006;11(2):93-100.

12. Wood MB, Murray PM. Current Concepts in the surgical management of brachial plexus injuries. Northeast Florida Medicine. 2006;57(3). Disponível em: <http:// www.researchgate.net/publication/228911777 Current Concepts_in_the_Surgical_Management_of_Brachial_ Plexus_Injuries>.

13. Haninec $P$, Sámal $F$, Tomás $R$, Houstava L, Dubovwý $P$. Direct repair (nerve grafting), neurotization, and end-to-side neurorrhaphy in the treatment of brachial plexus injury. J Neurosurg. 2007;106(3):391-9.

14. Siqueira MG, Martins RS. Surgical treatment of adult traumatic brachial plexus injuries: an overview. Arq Neuropsiquiatr. 2011;69(3):528-35.

15. Terzis JK, Vekris MD, Soucacos PN. Outcomes of brachial plexus reconstruction in 204 patients with devastating paralysis. Plast Reconstr Surg. 1999;104(5):1221-40.

16. Nagano A. Treatment of brachial plexus injury. J Orthop Sci. 1998;3(1):71-80 
17. Krishnan KG, Martin KD, Schackert G. Traumatic lesions of the brachial plexus: an analysis of outcomes in primary brachial plexus reconstruction and secondary functional arm reanimation. Neurosurgery. 2008;62(4):873-85.

18. Batista KT, Araújo HJ. Microcirurgia das lesões traumáticas de nervo periférico do membro superior. Rev Bras Cir Plast. 2010;25(4):708-14.

19. Sedel L. Management of supraclavicular lesions: clinical examination, surgical procedures and results. In: Terzis J, editor. Microreconstruction of nerve injuries. Philadelphia: Saunders; 1987. p. 385-92.

20. Leechavengvongs S, Witoonchart K, Uerpairojkit C, Thuvasethakul P, Malungpaishrope K. Combined nerve transfers for C5 and C6 brachial plexus avulsion injury. J Hand Surg Am. 2006;31(2):183-9.

21. Moiyadi AV, Devi BI, Nair KP. Brachial plexus injuries: outcome following neurotization with intercostal nerve. J Neurosurg. 2007;107(2):308-13.

22. Colbert SH, Mackinnon SE. Nerve transfers for brachial plexus reconstruction. Hand Clin. 2008;24(4):341-61.

23. Merle M, Becker C, Pankovic C, Bagot d'Arc M. Microsurgical repair of peripheral nerves and vessels using Tissucol. Clinical and experimental study. Rev Laryngol Otol Rhinol (Bord). 1987;108(1):13-4.

24. Samii A, Carvalho GA, Samii M. Brachial plexus injury: factors affecting functional outcome in spinal accessory nerve transfer for the restoration of elbow flexion. $J$ Neurosurg. 2003;98(2):307-12.

25. Ochiai $N$, Nagano A, Sugioka $H$, Hara T. Nerve grafting in brachial plexus injuries. Results of free grafts in 90 patients. J Bone Joint Surg Br. 1996;78(5):754-8.

26. Sulaiman OA, Kim DD, Burkett C, Kline DG. Nerve transfer surgery for adult brachial plexus injury: a 10-year experience at Louisiana State University. Neurosurgery. 2009;65(Suppl 4):A55-62.

27. Sedain G, Sharma MS, Sharma BS, Mahapatra AK. Outcome after delayed Oberlin transfer in brachial plexus injury. Neurosurgery. 2011;69(4):822-7.

28. Garg R, Merrell GA, Hillstrom HJ, Wolfe SW. Comparison of nerve transfers and nerve grafting for traumatic upper plexus palsy: a systematic review and analysis. J Bone Joint Surg Am. 2011;93(9):819-29.

29. Midha R. Nerve transfers for severe brachial plexus injuries: a review. Neurosurg Focus. 2004;16(5):E5.

30. Midha R. Emerging techniques for nerve repair: nerve transfers and nerve guidance tubes. Clin Neurosurg. 2006;53:185-90.

31. Amr SM, Essam AM, Abdel-Meguid AM, Kholeif AM, Moharram AN, El-Sadek R. Direct cord implantation in brachial plexus avulsions: revised technique using a single stage combined anterior (first) posterior (second) approach and end-to-side side-to-side grafting neurorrhaphy. J Brachial Plex Peripher Nerve Inj. 2009;4:8.
32. Sulaiman OA, Gordon T. Cellular and molecular interactions after peripheraland central injury. Rev Biomed. 2003;14:5162.

33. Leffert R. Brachial plexus injuries. Edinburgh: Churchill Livingstone; 1986.

34. Narakas AO. Brachial plexus injuries. In: McCarthy JG, editor. Plastic surgery: the hand. Philadelphia: Saunders; 1990. p. 4776-815.

35. Sulaiman OA, Gordon T. Transforming growth factor-beta and forskolin attenuate the adverse effects of long-term Schwann cell denervation on peripheral nerve regeneration in vivo. Glia. 2002;37(3):206-18.

36. Kandenwein JA, Kretschmer T, Engelhardt M, Richter HP, Antoniadis G. Surgical interventions for traumatic lesions of the brachial plexus: a retrospective study of 134 cases. J Neurosurg. 2005;103(4):614-21.

37. Souza FI, Saito M, Kimura LK, Mattar Júnior R, Zumiotti AV. Transferência do músculo tríceps para bíceps em pacientes com lesão crônica do tronco superior do plexo braquial. Rev Bras Ortop. 2010;45(4):409-12.

38. Tung TH, Mackinnon SE. Nerve transfers: indications, techniques, and outcomes. J Hand Surg Am. 2010;35(2):33241.

39. Oberlin C, Béal D, Leechavengvongs S, Salon A, Dauge MC, Sarcy JJ. Nerve transfer to biceps muscle using a part of ulnar nerve for C5-C6 avulsion of the brachial plexus: anatomical study and report of four cases. J Hand Surg Am. 1994;19(2):232-7.

40. Oberlin C, Durand S, Belheyar Z, Shafi M, David E, Asfazadourian $\mathrm{H}$. Nerve transfers in brachial plexus palsies. Chir Main. 2009;28(1):1-9.

41. Liverneaux PA, Diaz LC, Beaulieu JY, Durand S, Oberlin C. Preliminary results of double nerve transfer to restore elbow flexion in upper type brachial plexus palsies. Plast Reconstr Surg. 2006;117(3):915-9.

42. Birch R, Raji AR. Repair of median and ulnar nerves. Primary suture is best. J Bone Joint Surg Br. 1991;73(1):154-7.

43. Kotwal PP, Gupta V. Neglected tendon and nerve injuries of the hand. Clin Orthop Relat Res. 2005;431:66-71.

44. Martins RS, Siqueira MG, Heise CO, Teixeira MJ. Transfer of a fascicle from the posterior cord to the suprascapular nerve after injury of the upper roots of the brachial plexus: technical case report. Neurosurgery. 2009;65(4):E823-5.

\section{Endereço para correspondência}

Daniella Brito Rodrigues

Rua Silva Castro, 559, Guamá

66075-104 - Belém, PA, Brasil

Telefone: (91) 8815-7806

E-mail: britodaniella32@gmail.com 